\title{
Bending moment analysis of super large ore carrier based on graphical interface development
}

\author{
Liu Yongchao ${ }^{1}$, Liu Xiuwen ${ }^{1}$, Luan Xin ${ }^{1}$, and Xie Xingtao ${ }^{1}$ \\ ${ }^{1}$ Laboratory of Marine Dynamic Simulation \& Control, Dalian Maritime University, Dalian, China
}

\begin{abstract}
In order to improve the safety of shipping process, this paper designs a graphical user interface for calculating the static water bending moment of super large ore carrier. Due to the complexity of the actual loading conditions, the long hull girder and the mobility and sinking of cargo, the irregular distribution of gravity and buoyancy along the direction of the captain of the VLCC is prominent. In the actual loading process of ships, compartment loading is adopted in many cases, and the position where the maximum shear force and bending moment appear is generally not in the corresponding position. Traditional ship strength analysis methods can be divided into two kinds. One is to establish the ship's finite element model to calculate the ship's strength. The other is to calculate the ship's strength according to the simple beam bending theory. In this paper, a 360m VALEMAX ore carrier is taken as an example to calculate the local bending moment of the ship at a specific position by using QT software. Compared with the ship loading manual, the error of the program is small, and the program has guiding significance in the actual shipping process.
\end{abstract}

\section{Introduction}

The calculation of the shear force and bending moment of the ship is an important premise for the safety of the ship navigation and the foundation of the automatic loading of the ship. The calculation of static water shear and bending moment of large open deck ships such as bulk cargo and container is a mandatory requirement of SOLAS Convention. SOLAS Convention requires that all bulk carriers with a master of not less than $150 \mathrm{~m}$ and large open deck ships as defined in classification society specifications shall be equipped with corresponding computer software to calculate the static water shear and bending moment under various loading conditions. ${ }^{[1]}$ Even if the super large ship is optimized in hull strength, the probability of local shear and bending moment is higher than that of small ships due to the long captain. The probability of grounding and permanent damage of super large ships in the actual shipping process is also greatly improved due to the deformation of the hull.

At present, the ship strength calculation methods can be divided into two kinds: one is to establish the finite element model of the ship to calculate the ship's strength. The other is to calculate the ship strength according to the bending theory of simple beam. Gao Maojin uses the software of classification society of Norway and Max surf software of Australia to calculate the bending moment and shear force of the ship. The conclusion shows the approximate position of the maximum shear force and bending moment along the direction of the captain. ${ }^{[2]}$ Based on the three-dimensional STL model of hull and cabin, Liu Chunlei and Yinyong proposed a calculation method of floating state, static water shear force and bending moment of the ship under any loading condition, which made up for the deficiency of the conventional method $^{[1]}$.In the program module forcecalculator, zhangguangfa and liuyujun introduced the calculation method of checking the local stress and distribution of the total longitudinal bending moment of the hull girder in the launching analysis of large ships. ${ }^{[3]}$

In summary, the method of calculating ship strength based on simple beam is mainly based on the static hydraulic, tank capacity table and bond capacity curve provided by the ship design department. In this paper, the trapezoidal distribution method is used to design the graphical user interface to calculate the local bending moment of the ship.

\section{Traditional calculation methods of $s$ - hear force and bending moment}

\subsection{Related concepts of shear and bending mo- ment}

For shear forces, the absolute value of shear force (SF) on the cross section is equal to the difference between the gravity and buoyancy of the hull on either side of the profile. The allowable shear force value of large ships is usually given according to the calculation point, while the small ships usually only give the maximum shear allowable value. The allowable shear force is generally in the ship strength calculation. Ships with drainage capacity less than 10000 tons do not give allowable shear force at times, because the structure of this kind of

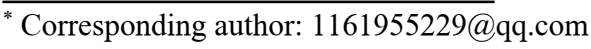


ship construction can meet the needs of ship shipping process.

For bending moment, the ship only gives the maximum allowable moment value, but some large ships will give the allowable bending moment value at each calculation point. The allowable bending moment value can be found in the ship strength calculation sheet. In the actual use, the allowable shear force and bending moment obtained in the ship strength calculation sheet are usually deducted according to the corrosion condition of the ship. Therefore, the actual local bending moment calculation results have a safe range.

The traditional definition of bending moment is that the moment of gravity on one side of the section is not equal to the moment of buoyancy to the section, and the moment of the hull shear force to the section makes up for the imbalance. This internal moment is called a moment. The curve of ship load distribution along the direction of the captain in still water is called the static water load curve. The one-time integral of the load along the direction of the master is called the hydrostatic shear curve. The first integral of the static water shear curve is the static water bending moment curve. ${ }^{[4-6]}$

\subsection{Calculation method of local still water bend- ing moment}

The two ends of the beam in equilibrium state are completely free in still water, so the shear force and bending moment at the end of bow and stern should be zero, that is, the shear force and bending moment are closed at the end. In most cases, because the load is approximately equal to the post midship position, the shear curve has the anti symmetric law. The zero point is close to the midship, while about 1/4 master has the maximum positive value or maximum negative value from the bow and stern end. Because the shear force at both ends is 0 , the slope of bending moment curve at both ends is zero. The integral of static water shear force and bending moment acting on any section of the hull beam along the captain $\mathrm{x}$ can be expressed as follows:

$$
\begin{gathered}
N_{s}(x)=\int_{0}^{x} q(x) d x \\
M_{s}(x)=\int_{0}^{x} N_{s}(x) d x=\int_{0}^{x} \int_{0}^{x} q(x) d x d x
\end{gathered}
$$

In the current code, the bending moment of a ship in still water is constant under a certain load condition. But in the actual sea conditions, the loading condition of the ship is not necessarily full load or ballast, sometimes there will be transshipment and other situations. In the actual loading and unloading of cargo, the influence of loading state on the static water bending moment is the main factor, so we should consider the negative influence of the static water bending moment on the hull load when calculating the cargo with fluidity. By checking the code for a specific ship, the load curve is directly reflected by the total longitudinal bending of the hull girder and the load distribution along the length of the ship. Each point on the curve is equal to the gravity and buoyancy difference of the corresponding position. In order to ensure the safe and economic navigation of the ship, this paper designs a program to calculate the shear force and bending moment for the super large ore carrier with complex loading conditions, which is used to guide the actual loading work of the ship.

\section{Analysis of shear force and bending moment of super large ship}

\subsection{Ships with uniform loading}

As shown in Figure 1, the shear force curve and bending moment curve of the ore carrier under uniform loading are shown. Because the local shear force is the derivative of the local bending moment, the shear diagram shows that the maximum shear force is $1 / 4$ of the ship's bow and stern. In the actual loading process, if the uniform loading method is adopted, because the loading method is relatively simple, it is enough to ensure that the bending moment value in the middle of the ship does not exceed the allowable value. However, the determination process of local shear force in the actual loading process is complex, and the maximum shear force of compartment loading is not necessarily in a fixed position. ${ }^{[7]}$

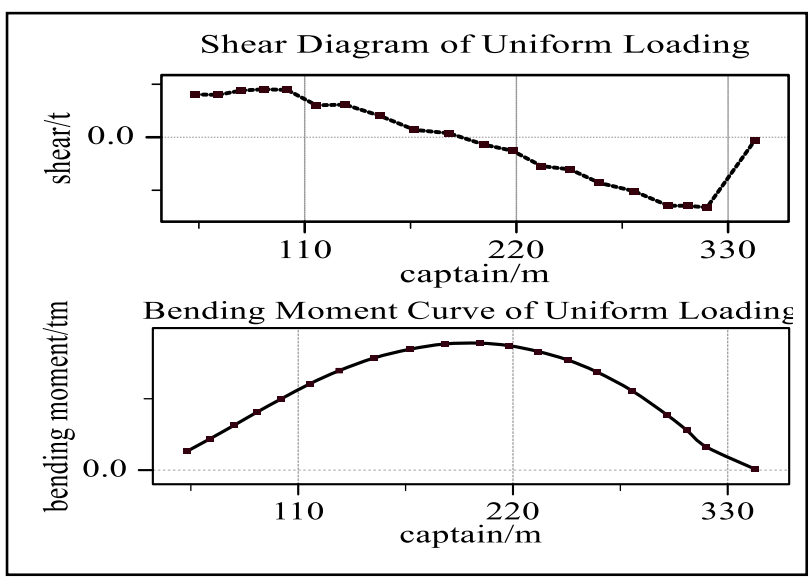

Fig.1. Shear force curve and bending moment curve of ship uniform loading

\subsection{Ship under compartment loading}

In the actual loading process, the compartment loading mode is often used for transportation, and the specific shear curve and bending moment curve are shown in Figure $2^{[8]}$. It is unreliable to estimate the maximum bending moment of a ship based on experience in the actual shipping process. Under normal conditions, the shear force curve can be obtained by integrating the load, and the bending moment curve can be obtained by integrating the shear force. But for the non-uniform compartment loading of super large ships, the calculation of the actual bending moment percentage and shear force percentage is a tedious process. In order to help check the local shear force or bending moment is too large in the actual loading and unloading and shipping process, this paper designs the following graphical user interface. 


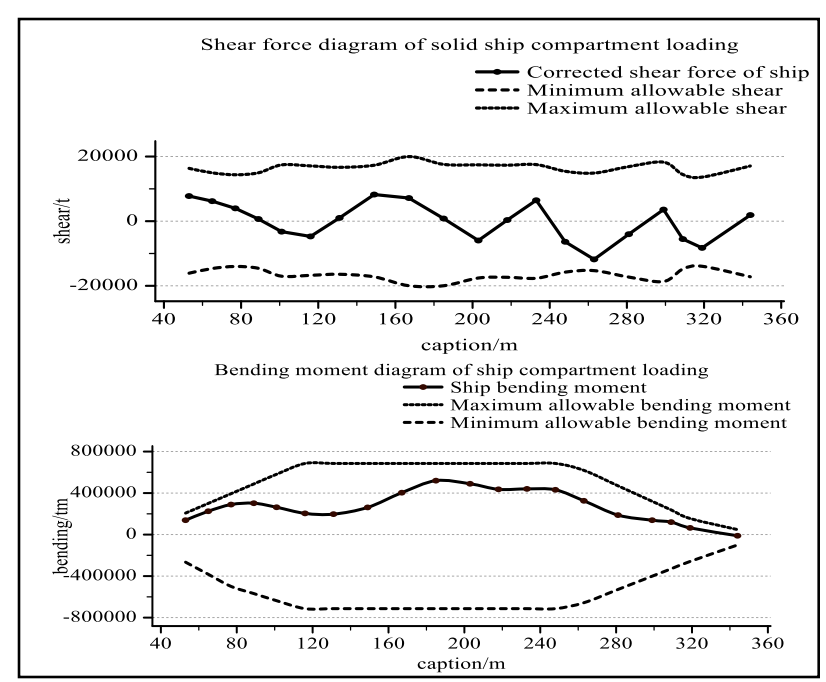

Fig.2. Curve of shear force and bending moment of ship compartment loading

\section{Algorithm implementation and exper- imentation and experimental results}

\subsection{Algorithm implementation and GUI}

When a super large ore carrier is loaded unevenly in compartments, due to the fluidity and sinking of the cargo, the ship's shear force at the corresponding position of the captain can not be reflected by the function expression. When calculating the bending moment, the trapezoidal method is used to approximate the integral of each section. As shown in Figure. 3, the shear segment curve $a_{1} a_{2}, a_{2} a_{3}, a_{3} a_{4} \cdots$ is a linear function, and the bending moment value at the corresponding position can be obtained by using the method of piecewise integral summation. A_ The calculation formula of bending moment at position $A_{2}$ is.

$$
A_{2}=A_{1}+\left(a_{1}+a_{2}\right) \cdot\left(A_{2}-A_{1}\right) / 2 .
$$

The bending moment at position $A_{3}$ is at $A_{2}$, The bending moment at position $\mathrm{A}_{4}$ is at $\mathrm{A}_{3}$, and then the bending moments at the following positions are superposed by this method.

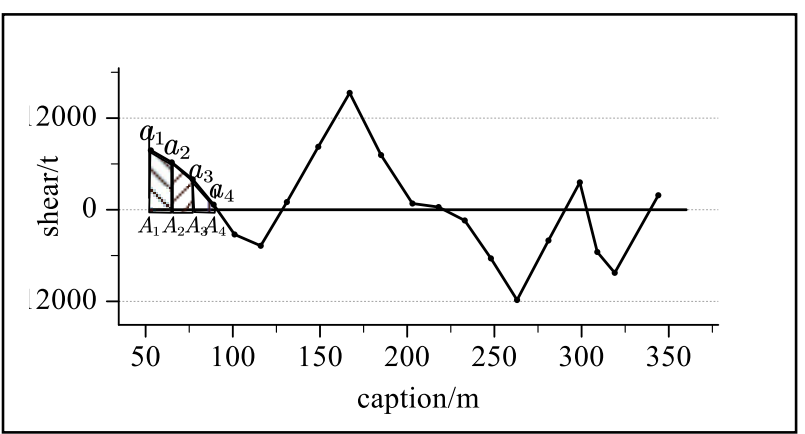

Fig.3. Real ship shear force partition diagram

In the actual loading process, the local shear force and bending moment of the ship should be less than the allowable shear force and bending moment of the corresponding position. This paper describes the corresponding procedure by taking the relation between shear force and bending moment as reference. The interface is designed by QT software, which takes the shear value modified by specific position as data input, and the data output is based on the relationship between theoretical moment value, theoretical moment value and longitudinal bulkhead modified bending moment ratio and allowable percentage of bending moment. The percentage of bending moment at different positions can be used to determine whether the load condition of the position needs further verification. For the bending moment value beyond the favorable and allowable range of hydrostatic bending moment, it can be more intuitive to show, and the captain or chief mate can take further measures.

The interface of initialization implementation is shown in Figure 4, which mainly involves lineedit, tablewidgt and qchartview controls. Input the shear force of the corresponding position of the ship in the lineedit input box, and click the calculation bending moment button to calculate the bending moment at the corresponding position and the allowable ratio of the corrected bending moment. The concrete and intuitive calculation results are shown in the ship moment table. Click the visualization button to reflect the calculated ship moment data and maximum bending moment in the curve of ship bending moment.

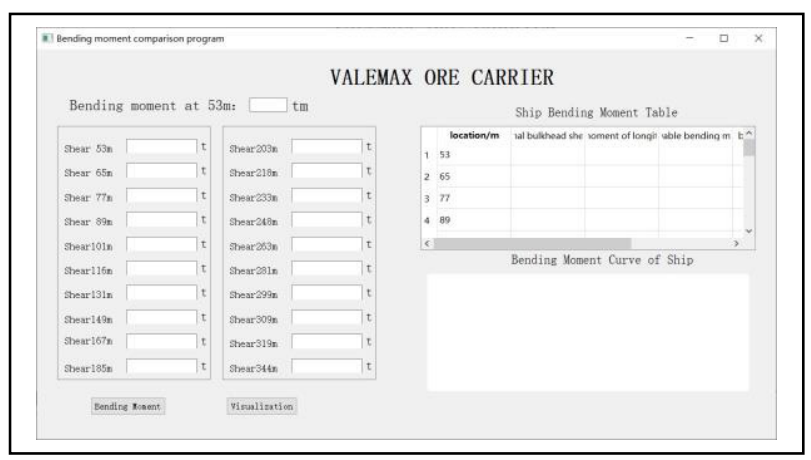

Fig.4. Initial calculation program for bending moment of VALEMAX ship

\subsection{Program Implementation}

According to the above program flow chart, the specific pseudo code is as follows:

//Add lineedit, tablewidgt, widgt control, and promote widgt control to qchartview type

//Design and click the slot function of "calculate bending moment" control

//The trapezoidal algorithm is used to calculate the bending moment of the ship with the data input by the user in lineedit, and the results are reflected in the table

//Design and click the "visualization" control slot function

//Add the data in the table to the qlineseries class, and bind the curve data to the qchart curve

The key codes for calculating bending moment by trapezoidal method and displaying the results in the table are as follows: 
int shear1=ui->lineEdit_2->text().toInt();

ui->tableWidget->setItem $(0,1$,new

QTableWidgetItem(QString::number(shear1)));

int shear2=ui->lineEdit_2->text().toInt();

ui->tableWidget->setItem $(1,1$, new

QTableWidgetItem(QString::number(shear2)));

int $\mathrm{x} 2=\mathrm{x} 1+($ shear $1+$ shear 2$) * 6$;

ui->tableWidget->setItem $(1,2$, new

QTableWidgetItem(QString::number(x2)));

The key code for visualizing the data in the table in the graph is as follows:

QLineSeries* linSeries=new QLineSeries(this);

QLineSeries* linSeries1=new QLineSeries(this);

QTableWidgetItem* $\mathrm{k} 1=$ ui->tableWidget->item $(0,2)$;

int $\mathrm{y} 1=\mathrm{k} 1->\operatorname{text}() \cdot \operatorname{toInt}()$;

linSeries->append $(53, y 1)$;

QChart* m_chart=new QChart();

m_chart->addSeries(linSeries);

m_chart->addSeries(linSeries 1$)$;

ui->widget_3->setChart(m_chart);

If the relationship between load and shear force is to be realized, the load in the program replaces the shear force, and the shear force replaces the bending moment.

As shown in Figure 5, some results of the program calculation are shown. The third column is the corrected bending moment value of longitudinal bulkhead calculated by integral. Through the relationship between theoretical bending moment and actual bending moment in the sixth column, it can be found that the overall ratio is about 1 , and the ratio near the stern fluctuates greatly. Considering the structure of stern type, the ship is loaded in five cargo tanks, and the ratio near the stern is not important. The results obtained by the iterative calculation of the piecewise trapezoidal method are not different from the actual values. During the shipping process, the shear force and bending moment of the corresponding position can be estimated through this program, and the points exceeding the allowable value in the specified value can be calculated accurately and the loading can be reasonably arranged.

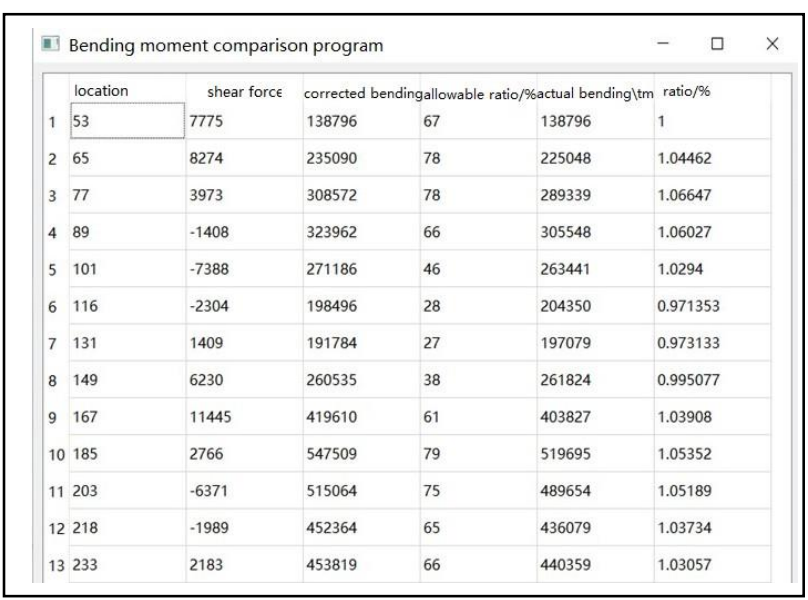

Fig. 5. Program calculation results

As shown in Figure 6, the user inputs the shear force at the corresponding position and clicks the "calculate bending moment" button, and the calculation results will be displayed in the bending moment table of the ship. On the basis of the bending moment results, click the "visualization" button, and the bending moment data in the table will be displayed on the ship bending moment curve. There is also a maximum bending moment curve in the figure, which is used to identify whether the bending moment value at the corresponding position exceeds the allowable range, and has the characteristics of simple visualization.

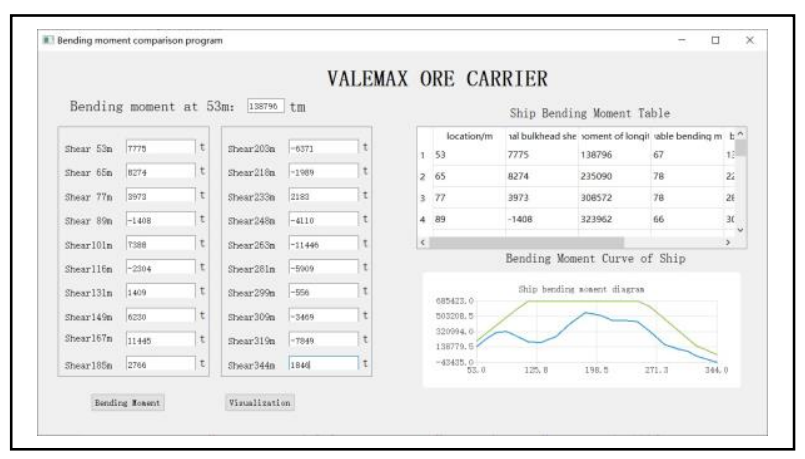

Fig.6. Visual graphic interface of bending moment for VALEMAX ore carrier

\subsection{Experimental Result}

As shown in Table 1, the calculation results of the program are compared with some experimental data in the code. It can be seen from the ratio relationship in the table that the overall ratio relationship of this trapezoidal calculation method is 1 . The ratio of the ship close to the stern fluctuates greatly, which may be related to the fact that most ships adopt the stern structure.

Table 1. Analysis table of program calculation results.

\begin{tabular}{|c|c|c|c|}
\hline Location/m & $\begin{array}{c}\text { Corrected } \\
\text { bending } \\
\text { moment of } \\
\text { longitudinal } \\
\text { bulkhead/tm }\end{array}$ & $\begin{array}{c}\text { Bending } \\
\text { moment } \\
\text { calculated by } \\
\text { program/tm }\end{array}$ & $\begin{array}{c}\text { Ratio } \\
\text { relation }\end{array}$ \\
\hline 53 & 138796 & 138796 & 1 \\
\hline 89 & 323962 & 305548 & 1.06 \\
\hline 116 & 198496 & 204350 & 0.97 \\
\hline 149 & 260535 & 261824 & 0.99 \\
\hline 185 & 547509 & 519695 & 1.05 \\
\hline 218 & 452364 & 436079 & 1.04 \\
\hline 24 & 439367 & 431494 & 1.02 \\
\hline 281 & 166502 & 187779 & 0.89 \\
\hline 299 & 108317 & 138649 & 0.78 \\
\hline
\end{tabular}

As shown in Figure 7, there is no significant difference between the side shell modified bending moment curve and longitudinal bulkhead modified bending moment curve at different captain positions and the actual ship bending moment curve, which has guiding significance in the actual shipping process. Considering the influence of wave, hull wear and other errors in the actual sea conditions, the local allowable load will fluctuate to a certain extent compared with the ex factory ship, so the actual influence of the error in the figure will not be 
great. The diagram is a comparison diagram of bending moment under load condition, and the local shear force can also be obtained by integral calculation of the program. The program has guiding significance in calculating local shear force and local bending moment.

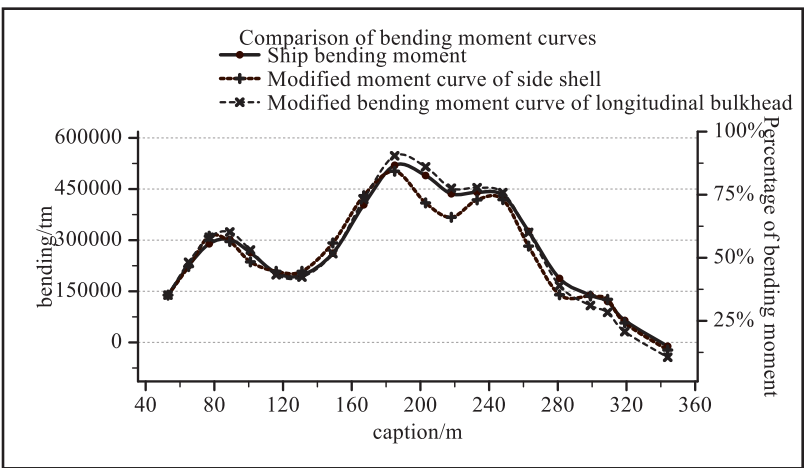

Fig.7. Comparison of bending moment curves

\section{Conclusion}

For the super large ship with non-uniform compartment loading, the maximum still water bending moment is not necessarily located near the middle of the ship, which is directly related to the loading state of the ship. Similarly, the maximum shear force of a ship may not be at the quarter of the ship's length from the fore and stern. In order to ensure the safe and economic navigation of the ship in the actual sea conditions, the ship loading should meet the allowable range and favorable range specified in the code.

The shear force and bending moment calculated by QT software segmented trapezoidal method have little difference with the actual value. In the actual shipping process, the graphical user interface is simple and visual, which has certain guidance.

This paper does not consider the calculation error caused by Hull wear and bulkhead correction. In the future, we can reduce the error by model, simulation and physical model.

\section{Acknowledgments}

Supported by the Ministry of industry and information technology (MIIT) zh [2018] No. 473 "intelligent ship comprehensive test and verification research" project; Special funds for basic scientific research in Central Universities (3132019312)

\section{References}

1. Liu Chunlei, Yin Yong. Calculation method of static water shear force and bending moment based on STL model [J], Journal of transportation engineering, 2017,17 (2): 73-82

2. Gao Maojin, Wu Jie. Direct calculation and analysis of longitudinal strength of $108 \mathrm{~m}$ deck cargo ship [J]. Jiangsu shipbuilding, 2014 (3) 12-13
3. Zhang Guangfa, Liu Yujun, Ji zhuoshang. General program design for longitudinal launching calculation of large ships based on Excel VBA [J]. Journal of Dalian University of technology, 2012,52 (01): 47-53

4. Xu Bangzhen, Tian Baijun. Ship freight [M]. Dalian: Dalian Maritime University Press, 2011

5. Du Jiali, Jiang Hua. Principles of communication [M] Dalian: Dalian Maritime University Press, 2016

6. Wu Chunsheng, Tian Baijun, Qiu Wenchang. Maritime cargo transportation [M]. Beijing: People's Communications Press, 2012

7. Liu Yongchao, Cui Jianhui. Discussion on improvement of average draught of super large dry bulk carrier [J], Tianjin navigation 2021 (4): 1-4

8. Huang min. calculation and analysis of longitudinal strength of 3000dwt deck cargo ship [J]. China Shipping (second half), 2013,12 (11): 28-29 